\title{
First successful case of percutaneous transabdominal thoracic duct embolization (PTTDE) for chylous ascites resulting from laparoscopic gastric cancer surgery
}

\author{
Hideyuki Yokokawa ${ }^{1,2}$ (1) Takao Katsube ${ }^{1} \cdot$ Miki Miyazawa $^{1} \cdot$ Ryohei Nishiguchi $^{1} \cdot$ Shinichi Asaka ${ }^{1}$. \\ Kentaro Yamaguchi ${ }^{1}$ - Minoru Murayama ${ }^{1}$. Kotaro Kuhara ${ }^{1}$. Takebumi Usui ${ }^{1}$. Hajime Yokomizo ${ }^{1}$. \\ Kazuhiko Yoshimatsu ${ }^{1,2} \cdot$ Takeshi Shimakawa $^{1} \cdot$ Shunichi Shiozawa ${ }^{1}$
}

Received: 23 September 2020 / Accepted: 6 January 2021 / Published online: 23 January 2021

(c) The Author(s) 2021

\begin{abstract}
A 61-year-old woman underwent laparoscopy-assisted distal gastrectomy (LADG) with extragastric lymph node dissection (D2). Two months later, she was readmitted to hospital to be treated for chylous ascites. Oral intake was discontinued and total parenteral nutrition started, but increasing body weight and decreasing serum albumin concentration was not controllable. Percutaneous transabdominal thoracic duct embolization (PTTDE) was performed on the 8th day after the readmission. Five days after PTTDE, oral intake was resumed. Seventeen days after PTTDE, the patient was discharged without recurrence of ascites. She has remained asymptomatic. We describe here the first patient with chylous ascites two months after LADG with D2 dissection for early gastric cancer who was successfully treated by PTTDE.
\end{abstract}

Keywords Chylous ascites $\cdot$ Stomach neoplasms $\cdot$ Gastrectomy

\section{Introduction}

Laparoscopy-assisted distal gastrectomy has been shown feasible for early gastric cancer [1-5]. As primary complications after LADG, anastomotic leakage (1.7\%), pancreatic fistula (1.7\%), intraabdominal abscess (1.5\%), delayed gastric emptying $(0.7 \%)$, postoperative hemorrhage $(0.4 \%)$, wound infection $(0.4 \%)$, bowel obstruction $(0.2 \%)$, and pneumonia $(0.2 \%)$ have been reported $[4,6]$. In laparoscopic gastrectomy with D1-2 dissection, the incidence of chylous ascites is markedly low at $0.3-0.7 \%$ [7].

Here, we present our first patient with chylous ascites two months after LADG with D2 dissection for early gastric cancer who was successfully treated with percutaneous transabdominal thoracic duct embolization (PTTDE).

Hideyuki Yokokawa

yokokawa.hideyuki@twmu.ac.jp

1 Department of Surgery, Tokyo Women's Medical University, Medical Center East, 2-1-10 Nishiogu, Arakawa, Tokyo 116-8567, Japan

2 Department of Surgery, Saitama-ken Saiseikai Kurihashi Hospital, 714-6 Koemon, Kuki, Saitama 349-1105, Japan

\section{Case report}

A 61-year-old woman was admitted to our hospital for treatment of gastric cancer. Preoperative examination showed that the tumor was located in the middle part of the stomach and had invaded the muscularis propria (MP) but without lymph node metastasis. She underwent LADG with extragastric lymph node dissection (D2) [8] using laparoscopic ultrasonic shears (Harmonic Scalpel; Ethicon Endo-Surgery, Cincinnati, OH, USA). Billroth I gastroduodenostomy was performed, and a drain was placed under the left lobe of the liver from the right upper abdomen. On postoperative day 2 , the patient was allowed oral intake of food. The drain was removed at postoperative day 3 . The postoperative course was uneventful and she was discharged at postoperative day 14. Histological tumor findings were M, Less, Type 2, $50 \times 20 \mathrm{~mm}$, por $>$ sig, pT1b (SM2), pN1 (2/47), pStage IB [9].

Two months after LADG, she was readmitted to hospital because of abdominal distension. Her weight was $55.0 \mathrm{~kg}$, a gain of $0.8 \mathrm{~kg}$ since discharge. Physical examination revealed increasing abdominal girth and bilateral leg edema. The serum albumin concentration was $3.0 \mathrm{~g} / \mathrm{dL}$, showing hypoalbuminemia. Ultrasound examination confirmed 
diffuse ascites. A percutaneous peritoneal drainage catheter was placed in the peritoneal cavity under ultrasonographic imaging, and $7100 \mathrm{~mL}$ of white-milky fluid was drained which had a high triglyceride level of $1320 \mathrm{mg} / \mathrm{dL}$. Cytological examination of the ascites showed it to be class IIb. Bacterial culture was negative. We therefore made a diagnosis of chylous ascites.

Although a fat-restricted diet was initiated, abdominal girth gradually increased. Oral intake was discontinued and total parenteral nutrition started on the 5th day, but increasing body weight and decreasing serum albumin concentration was not controlled. Because no improvement was observed, PTTDE was performed on the 8th day.

\section{PTTDE}

Ultrasound-guided access to the left inguinal lymph nodes was achieved with a $26 \mathrm{G}$ needle. Lipiodol was slowly administered to the lymph nodes. Fluoroscopic imaging showed an accumulation of Lipiodol in the left lumbar lymphatic channels (Fig. 1) and the thoracic duct. Fluoroscopic imaging indicated the leakage site to be in the upper abdominal thoracic duct (Fig. 2).

Percutaneous transabdominal thoracic duct cannulation was performed using a $21 \mathrm{G}$ Chiba needle at the level of the navel under fluoroscopic guidance, and a V-18 peripheral guidewire was used to probe for the duct. Once the thoracic duct had been accessed, a microcatheter (Prominent Raptor) was passed over the guide wire into the upper thoracic duct. Thoracic duct embolization was then performed. First, microcoils (AZUR CX $6 \mathrm{~mm}-20 \mathrm{~cm}$ and $5 \mathrm{~mm}-15 \mathrm{~cm}$ ) were positioned to provide a matrix for glue polymerization on the thoracic part of the thoracic duct, after which 20\% n-butyl cyanoacrylate (NBCA) diluted with Lipiodol was used for embolization (Fig. 3). Abdominal CT scan obtained immediately after lymphangiography and PTTDE showed contrast pooling between

Fig. 1 Fluoroscopic image showing injection of Lipiodol through the left inguinal lymph nodes (arrow)

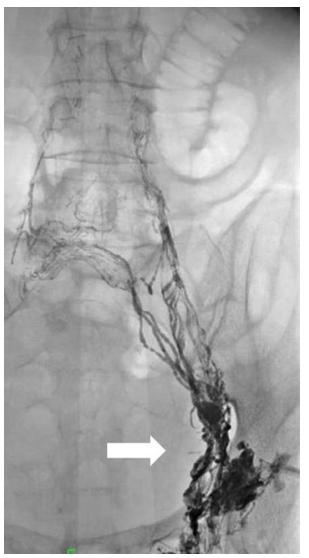

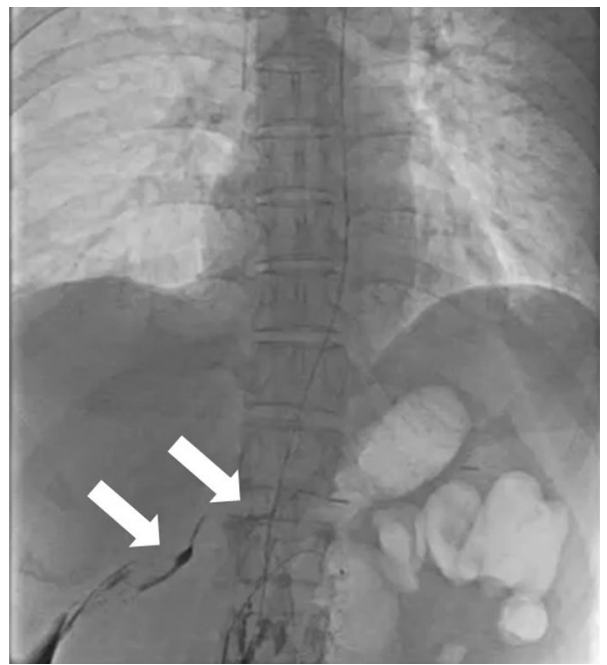

Fig. 2 Lymphangiographic image demonstrating contrast extravasation (arrows)

the abdominal aorta and the pancreas head and contrast extravasations accumulating at Morrison's pouch (Fig. 4).

Two days after thoracic duct embolization, her body weight showed a progressive decrease and inversely, her serum albumin concentration increased. Five days after thoracic duct embolization, oral intake was resumed and on the 24th day (17 days after thoracic duct embolization), she was discharged without recurrence of ascites (Fig. 5). She has remained asymptomatic.

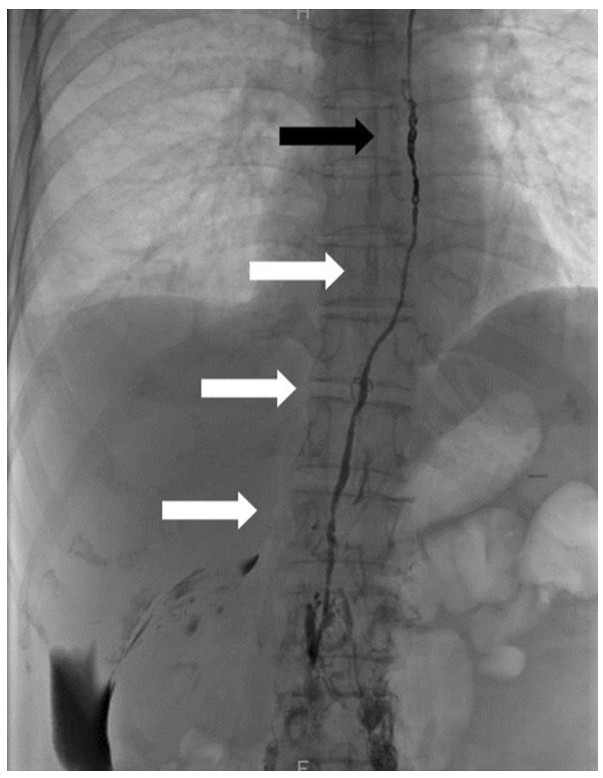

Fig. 3 Fluoroscopic image of the embolization with NBCA glue (white arrows) and microcoils (black arrow) 

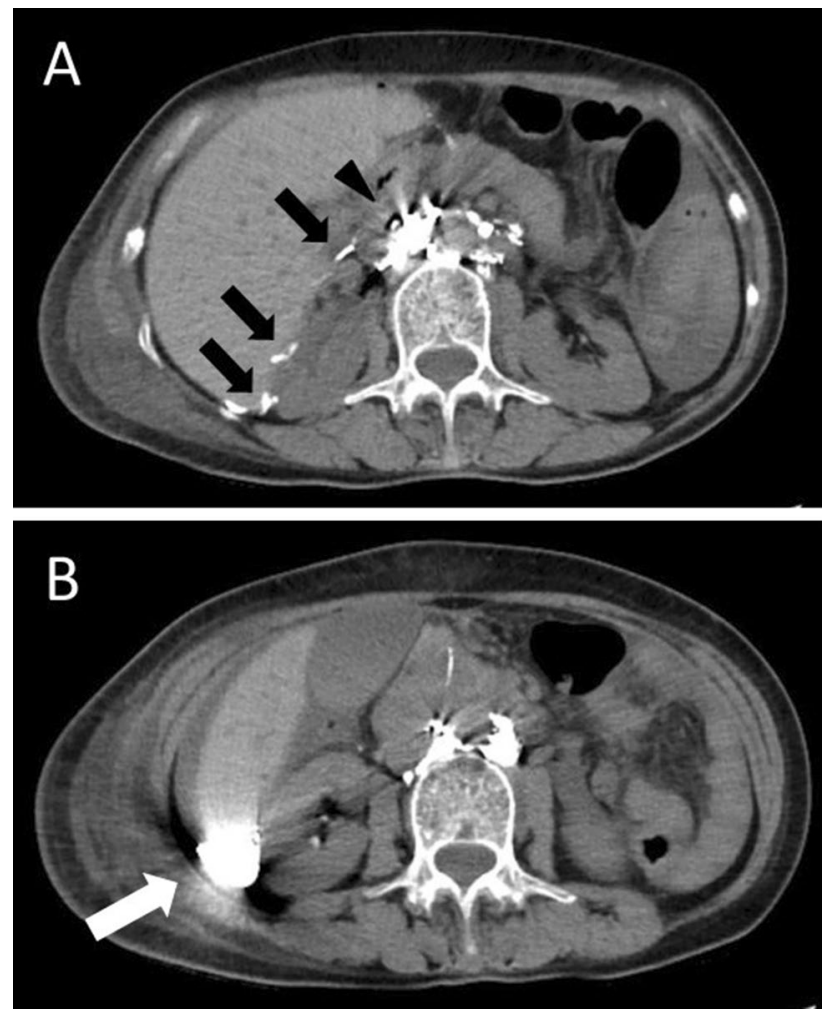

Fig. 4 Abdominal CT scan obtained immediately after lymphangiography and PTTDE. a Contrast pooling between the abdominal aorta and the pancreas head (black arrow head) and contrast extravasations (black arrows). b The accumulation of contrast at Morrison's pouch (white arrow)

\section{Discussion}

Chylous ascites after surgery for abdominal malignant tumors is caused by damage to the cisterna chyli, thoracic duct, lymphatic vessels, or stasis of lymphatic system; however, the chief cause is considered to be direct damage to the lymphatic systems resulting from the surgical operation [10]. The incidence of chylous ascites due to gastric cancer surgery is reported to be $6.3-11.8 \%$ for periaortic lymph node dissection and $1.99 \%$ for D1-2 lymph node dissection $[11,12]$. In laparoscopic gastrectomy with D1-2 dissection, the incidence of chylous ascites is notably low, at $0.3-0.7 \%$ [7]. The cause of chylous ascites after gastric cancer surgery without periaortic lymph node dissection can be due to anatomical variation of the abdominal lymphatic plexus and cisterna chyli $[11,13,14]$. As risk factors for chylous ascites after major abdominal surgery, some factors were listed [7]. In our case, female sex and number of lymph node resection (the median total number of lymph nodes harvested in patients developing a chyle leak was 18) matched the risk factors [7].

Although there are no consistent diagnostic criteria, in most of the literature, a clinically significant postoperative chylous ascites is best defined as the appearance of milky and nonpurulent fluid in the drainage tube with a triglyceride concentration exceeding $110 \mathrm{mg} / \mathrm{dL}$ and a daily minimum volume of $200 \mathrm{~mL}$ [7].

The onset of chylous ascites tends to occur from the fifth to the twelfth days after surgery, when the volume of meals and lymph flow start to increase [15]. There are reports similar to that of our case describing the late onset of chylous ascites, occurring a few weeks to a few months after surgery $[11,16,17]$, and lymph flow stasis is proposed to be
Fig. 5 Clinical course: Changes in serum albumin value (Alb) and body weight after hospital admission

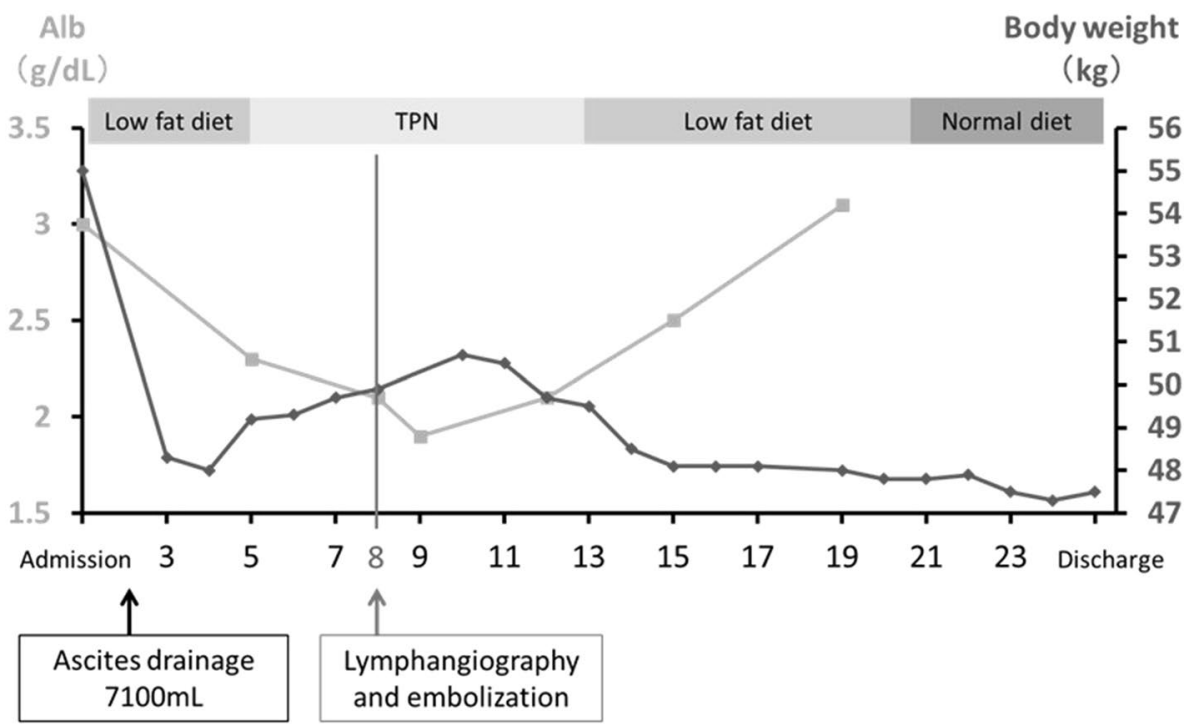


a cause [16-18]. It is reported that the incidence of stasis chylous ascites is increased by ligation of the lymph nodes around the celiac or the superior mesenteric artery [18] and extensive fibrosis of periaortic lymph nodes after preoperative chemotherapy [16]; however, there are no reports of stasis chylous ascites after laparoscopic gastrectomy. In our case, laparoscopic ultrasonic shears that enable coagulotomy of lymphatic vessels [19] were used. It is possible that late onset of stasis chylous ascites occurred as a result of complete obstruction of lymphatic flow by the USAD.

The first choice for postoperative chylous ascites treatment is conservative treatment, which includes fat-restricted diets or fasting, and high calorie infusions for the purpose of reducing lymph. Approximately, $70-80 \%$ of cases show remission within 2-6 weeks of treatment $[7,20]$. Some studies report octreotide, a somatostatin analog, to be effective $[7,20,21]$; however, surgical treatment is considered if conservative treatment is not effective, such as when 1 . There is no improvement after two weeks or longer with conservative treatment; 2. Lymphatic leakage of $1000 \mathrm{ml} /$ day or more continues for a week or longer; and 3. Nutritional conditions deteriorate [20].

We applied PTTDE with lymphangiography eight days after starting conservative treatment. Only two cases of lymphangiography are reported for chylous ascites after gastric cancer surgery [22]. We also performed a lymphangiography by puncturing the inguinal lymph node under echo guidance. Fluoroscopic imaging suggested the leakage site to be in the upper abdominal thoracic duct. Lymphangiography is regarded as a simple and minimally invasive diagnostic method [23]. It is also reported that, as an effect of the embolization of Lipiodol, $89 \%$ of postoperative chylous ascites cases were improved by lymphography alone [23-25]. Here, we propose another hypothesis of our late onset of chylous ascites from the results of lymphangiography and followed abdominal CT scan. Those examinations revealed that leaked contrast was accumulating at Morrison's pouch, which means there is the possibility that the drain placed under left lobe of the liver after LADG could not work well, though chylous ascites had occurred already before discharge. A case who developed chylous ascites 25 days after laparoscopic total gastrectomy with D1 plus dissection for gastric cancer was reported [26]. In the case, as a result of reviewing the surgical video, damage to the lymphatic vessels was confirmed during No. 8a lymph node dissection. We also reviewed our surgical video and found no lymphatic damage on the video, but chylous ascites could have already developed on the early postoperative days and get symptomatic over time.

On the other hand, the effectiveness of percutaneous catheterization and embolization of the thoracic duct (PCETD) against refractory chylous pleural effusion were reported in the United States in 1998, and since then, its effectiveness against refractory chylous ascites has also been shown [27-29]. Although complications of pulmonary embolism (several cases), pedal edema (7\%), and chronic diarrhea (8.7\%) due to embolization material are also reported [28, $29], 82 \%$ of chylous ascites cases in which a lymphatic leakage section was identified by lymphangiography are reported to be improved by lymphatic embolization [29].

This is the first case of successful treatment by PTTDE of chylous ascites caused by laparoscopic gastric cancer surgery. No complications were observed after lymphangiography or PTTDE. Lymphography and PTTDE for postoperative chylous ascites proved to be safe and effective treatments.

Acknowledgments We thank Yoshiaki Katada for his assistance with PTTDE for chylous ascites.

\section{Compliance with ethical standards}

Conflict of interest We declare that none of the physicians have any conflicts of interest.

Research involving human participants and/or animals Formal consent is not required for this type of study. This article does not contain any studies with human participants or animals performed by any of the authors.

Informed consent Informed consent was obtained from the individual who participated in the study.

Open Access This article is licensed under a Creative Commons Attribution 4.0 International License, which permits use, sharing, adaptation, distribution and reproduction in any medium or format, as long as you give appropriate credit to the original author(s) and the source, provide a link to the Creative Commons licence, and indicate if changes were made. The images or other third party material in this article are included in the article's Creative Commons licence, unless indicated otherwise in a credit line to the material. If material is not included in the article's Creative Commons licence and your intended use is not permitted by statutory regulation or exceeds the permitted use, you will need to obtain permission directly from the copyright holder. To view a copy of this licence, visit http://creativecommons.org/licenses/by/4.0/.

\section{References}

1. Kitano S, Iso Y, Moriyama M, Sugimachi K (1994) Laparoscopyassisted Billroth I gastrectomy. Surg Laparosc Endosc 4:146-148

2. Fujiwara M, Kodera Y, Kasai Y et al (2003) Laparoscopy-assisted distal gastrectomy with systemic lymph node dissection for early gastric carcinoma: a review of 43 cases. J Am Coll Surg 196:75-81

3. Yoshikawa T, Cho H, Rino Y et al (2013) A prospective feasibility and safety study of laparoscopy-assisted distal gastrectomy for clinical stage I gastric cancer initiated by surgeons with much experience of open gastrectomy and laparoscopic surgery. Gastric Cancer 16:126-132

4. Katai H, Mizusawa J, Katayama H et al (2017) Short-term surgical outcomes from a phase III study of laparoscopy-assisted 
versus open distal gastrectomy with nodal dissection for clinical stage IA/IB gastric cancer: Japan Clinical Oncology Group Study JCOG0912. Gastric Cancer 20:699-708

5. Hiki N, Katai H, Miyazawa J et al (2018) Long-term outcomes of laparoscopy-assisted distal gastrectomy with suprapancreatic nodal dissection for clinical Stage I gastric cancer: a multicenter phase II trial (JCOG0703). Gastric Cancer 21:155-161

6. Katai H, Sasako M, Fukuda $\mathrm{H}$ et al (2010) Safety and feasibility of laparoscopy-assisted distal gastrectomy with suprapancreatic nodal dissection for clinical stage I gastric cancer: a multicenter phase II trial (JCOG 0703). Gastric Cancer 13:238-244

7. Maximilian W, Jan GD, Martin KA et al (2016) Treatment options for chylous ascites after major abdominal surgery: a systematic review. Am J Surg 211:206-213

8. Japanese Gastric Cancer Association (2020) Japanese gastric cancer treatment guidelines 2018 (5th edition). Gastric Cancer. https ://doi.org/10.1007/s10120-020-01042-y

9. Japanese Gastric Cancer Association (2011) Japanese classification of gastric carcinoma. 3rd English edition. Gastric Cancer $14: 101-112$

10. Kelly ML, Butt HR (1960) Chylous ascites: an analysis of its etiology. Gastroenterology 39:161-170

11. Hüseyin Ç, Kamuran CD, Ebubekir G et al (2015) Late onset of chylous ascites following distal gastrectomy with D1 (+) dissection for gastric cancer: a case repot. Dicle Med J 42:514-517

12. Yol S, Bostanci EB, Ozogul Y et al (2005) A rare complication of D3 dissection for gastric carcinoma: chyloperitoneum. Gastric Cancer 8:35-38

13. Yamada T, Jin Y, Hasuo K et al (2013) Chylorrhea following laparoscopy assisted distal gastrectomy with D1+ dissection for early gastric cancer. A case report. Int J Surg Case Rep 3:1173-1175

14. Griniatsos J, Dimitriou N, Kyriaki D et al (2010) Chylorrhea complicating D2+a gastrectomy: review of the literature and clarification of terminology apropos one case. Chin Med J 123:2279-2283

15. Kaas R, Rustman LD, Zoetmulder FAN (2001) Chylous ascites after oncological abdominal surgery: incidence and treatment. Eur J Surg Oncol 27:187-189

16. Kawamura H, Tanioka T, Funakoshi T et al (2011) Two cases of delayed chyloperitoneum after gastrectomy. J Jpn Surg Assoc 72:1122-1125

17. Miyazaki S, Kimura Y, Fujitani K et al (2015) A case report of chylous ascites after one month of radial gastrectomy. J Jpn Coll Surg 40:858-862
18. Endo M, Maruyama K, Kinoshita T et al (1994) Chylous ascites after extended lymph node dissection for gastric cancer. Jpn J Gastroenterol Surg 27:917-921

19. Nakayama H, Ito H, Kato Y, Tsuboi M (2009) Ultrasonic scalpel for sealing of the thoracic duct: evaluation of effectiveness in an animal model. Interact Cardiovasc Thorac Surg 9:399-401

20. Shulan LV, Qing W, Wanqiu Z et al (2017) A review of the postoperative lymphatic leakage. Oncotarget 40:69062-69075

21. Kanda H, Kadono J, Ishizaki N et al (2011) The successful use of Octreotide in refractory chylous ascites: two case reports. J Jpn Surg Assoc 72:2139-2142

22. Kawasaki R, Sugimoto K, Fujii M et al (2013) Therapeutic effectiveness of diagnostic lymphangiography for refractory postoperative chylothorax and chylous ascites: correlation with radiologic findings and preceding medical treatment. J Vasc Interv Radiol 201:659-666

23. Hanada K, Hata H, Otani T et al (2016) A case of chylothorax after esophagectomy for esophageal cancer performed by thoracoscopic thoracic duct ligation in which lymphangiography by inguinal lymph node puncture was useful. J Jpn Surg Assoc 77:322-327

24. Yasui H, Makino Y, Mikami S et al (2017) Lymphangiogram for chylous ascites due to lung adenocarcinoma. AJRS 6:274-277

25. Matsumoto T, Yamaguchi T, Kato T et al (2009) The effectiveness of lymphangiography as a treatment method for various chyle leakages. Br J Radiol 82:286-290

26. Suzui S, Kinoshita T, Shibasaki H et al (2016) Three cases of chylous ascites after laparoscopic radical gastrectomy (in Japanese). Rinsho Geka J Clin Surg 71:1153-1157

27. Cope C (1998) Diagnosis and treatment of postoperative chyle leakage via percutaneous transabdominal catheterization of the cisterna chyli: a preliminary study. J Vasc Interv Radiol 9:727-734

28. Chen E, Itkin M (2011) Thoracic duct embolization for chylous leaks. Semin Intervent Radiol 28:63-74

29. Nadolski GJ, Chauhan NR, Itkin M (2018) Lymphangiography and lymphatic embolization for the treatment of refractory chylous ascites. Cardiovasc Intervent Radiol 41:415-423

Publisher's Note Springer Nature remains neutral with regard to jurisdictional claims in published maps and institutional affiliations. 